\title{
Cosmological Shock Waves
}

\author{
A.M. Bykov • K. Dolag • F. Durret
}

Received: 17 September 2007 / Accepted: 15 October 2007 / Published online: 12 February 2008 (C) Springer Science+Business Media B.V. 2008

\begin{abstract}
Large-scale structure formation, accretion and merging processes, AGN activity produce cosmological gas shocks. The shocks convert a fraction of the energy of gravitationally accelerated flows to internal energy of the gas. Being the main gas-heating agent, cosmological shocks could amplify magnetic fields and accelerate energetic particles via the multi-fluid plasma relaxation processes. We first discuss the basic properties of standard single-fluid shocks. Cosmological plasma shocks are expected to be collisionless. We then review the plasma processes responsible for the microscopic structure of collisionless shocks. A tiny fraction of the particles crossing the shock is injected into the non-thermal energetic component that could get a substantial part of the ram pressure power dissipated at the shock. The energetic particles penetrate deep into the shock upstream producing an extended shock precursor. Scaling relations for postshock ion temperature and entropy as functions of shock velocity in strong collisionless multi-fluid shocks are discussed. We show that the multi-fluid nature of collisionless shocks results in excessive gas compression, energetic particle acceleration, precursor gas heating, magnetic field amplification and non-thermal emission. Multi-fluid shocks provide a reduced gas entropy production and could also modify the observable thermodynamic scaling relations for clusters of galaxies.
\end{abstract}

Keywords Large scale structure of universe - Shock waves - Acceleration of particles · $\mathrm{X}$-rays: galaxies: clusters

\footnotetext{
A.M. Bykov ( $\square)$

A.F. Ioffe Institute of Physics and Technology, St. Petersburg 194021, Russia

e-mail: byk@astro.ioffe.ru

K. Dolag

Max-Planck-Institut für Astrophysik, P.O. Box 1317, 85741 Garching, Germany

e-mail:kdolag@mpa-garching.mpg.de

F. Durret

Institut d'Astrophysique de Paris, CNRS, Université Pierre et Marie Curie, 98bis Bd Arago, 75014 Paris, France

e-mail: durret@iap.fr
} 


\section{Introduction}

The observed large scale structure of the Universe is thought to be due to the gravitational growth of density fluctuations in the post-inflation era. In this model, the evolving cosmic web is governed by non-linear gravitational growth of the initially weak density fluctuations in the dark energy dominated cosmology. The web is traced by a tiny fraction of luminous baryonic matter. Cosmological shock waves are an essential and often the only way to power the luminous matter by converting a fraction of gravitational power to thermal and nonthermal emissions of baryonic/leptonic matter.

At high redshifts $(z>1100)$ the pre-galactic medium was hot, relatively dense, ionised, with a substantial pressure of radiation. The cosmic microwave background (CMB) observations constrain the amplitudes of density inhomogeneities to be very small at the last scattering redshift $z \sim 1000$. Strong non-linear shocks are therefore unlikely at that stage. The universe expands, the matter cools, and eventually recombines, being mostly in neutral phase during the "dark ages" of the universe. At some redshift, $6<z<14$, hydrogen in the universe is reionised, likely due to UV radiation from the first luminous objects, leaving the intergalactic medium (IGM) highly reionised (see e.g., Fan et al. (2006) for a recent review). The reionisation indicates the formation of the first luminous objects at the end of the "dark ages", either star-forming galaxies or Active Galactic Nuclei (AGN). The compact luminous objects with an enormous energy release would have launched strong (in some cases, relativistic) shock waves in the local vicinity of the energetic sources. At the same evolution stage, formation of strong density inhomogeneities in the cosmic structure occurs. Since then the non-linear dynamical flows in the vicinity of density inhomogeneities would have created large scale cosmic structure shocks of modest strength, thus heating the baryonic matter and simultaneously producing highly non-equilibrium energetic particle distributions, magnetic fields and electromagnetic emission.

Most of the diffuse X-ray emitting matter was likely heated by cosmological shocks of different scales. Accretion and merging processes produce large-scale gas shocks. Simulations of structure formation in the Universe predict that in the present epoch about $40 \%$ of the normal baryonic matter is in the Warm-Hot Intergalactic Medium (WHIM) at overdensities $\delta \sim 5-10$ (e.g., Cen and Ostriker 1999; Davé et al. 2001). The WHIM is likely shock-heated to temperatures of $10^{5}-10^{7} \mathrm{~K}$ during the continuous non-linear structure evolution and star-formation processes.

The statistics of cosmological shocks in the large-scale structure of the Universe were simulated in the context of the $\Lambda \mathrm{CDM}$-cosmology using PM/Eulerian adiabatic hydrodynamic codes (e.g., Miniati et al. 2000; Ryu et al. 2003; Kang et al. 2007) and more recently with a smoothed particle hydrodynamic code by Pfrommer et al. (2006). They identified two main populations of cosmological shocks: $(i)$ high Mach number "external" shocks due to accretion of cold gas on gravitationally attracting nodes, and (ii) moderate Mach number $\left(2 \leq \mathcal{M}_{\mathrm{s}} \leq 4\right)$ "internal" shocks. The shocks are due to supersonic flows induced by relaxing dark matter substructures in relatively hot, already shocked, gas. The internal shocks were found by Ryu et al. (2003) to be most important in energy dissipation providing intercluster medium (ICM) heating, and they were suggested by Bykov et al. (2000) to be the likely sources of non-thermal emission in clusters of galaxies.

Hydrodynamical codes deal with N-body CDM and single-fluid gas dynamics. However, if a strong accretion shock is multi-fluid, providing reduced post-shock ion temperature and entropy, then the internal shocks could have systematically higher Mach numbers.

Space plasma shocks are expected to be collisionless. Cosmological shocks, being the main gas-heating agent, generate turbulent magnetic fields and accelerate energetic particles 
via collisionless multi-fluid plasma relaxation processes thus producing non-thermal components. The presence of these non-thermal components may affect the global dynamics of clusters of galaxies (Ostriker et al. 2005) and the $\sigma_{\mathrm{v}}-T, M-T, L_{\mathrm{X}}-T$ scaling relations (Bykov 2005). Detailed discussion of the cosmological simulations of the scaling relations with account of only thermal components can be found in Borgani et al. (2008-Chap. 13, this issue).

In Sect. 2 we discuss the basic features of the standard collisional shocks. The main part of the review is devoted to physical properties of cosmological shocks with an accent on collisionless shocks and associated non-thermal components. In Sect. 5 we discuss the most important features of multi-fluid collisionless shocks in the cosmological context including the effects of reduced entropy production, energetic particle acceleration and magnetic field amplification in the shocks.

\section{Single-Fluid MHD-Shocks}

Shock waves are usually considered as a sharp transition between a macroscopic supersonic (and super-Alfvénic) upstream flow (state 1) and slowed down to a subsonic velocity downstream flow (state 2), providing a mass flow $j_{n}$ through the shock surface. It is assumed that a gas particle (or an elementary macroscopic fluid cell) is at any instant of time in the local thermodynamic equilibrium state corresponding to the instantaneous values of the macroscopic parameters. The Maxwellian distribution of all species is ensured after a few molecular (or Coulomb) collisions have occurred. The macroscopic parameters characterising the state of the gas, such as density, specific internal energy, or temperature, change slowly in comparison with the rates of the relaxation processes leading to thermodynamic equilibrium. We consider here a single-fluid plasma model assuming complete electron-ion relaxation. Under these conditions, in a frame moving with the shock front, with the matter flux across the shock surface $j_{n} \neq 0$, the conservation laws for mass (in non-relativistic flows), momentum and energy can be written as follows:

$$
\begin{gathered}
j_{n}\left[\frac{\mathbf{B}_{\mathbf{t}}}{\rho}\right]=B_{n}\left[\mathbf{u}_{\mathbf{t}}\right], \\
j_{n}\left[\mathbf{u}_{\mathbf{t}}\right]=\frac{B_{n}}{4 \pi j_{n}}\left[\mathbf{B}_{\mathbf{t}}\right], \\
{\left[\frac{j_{n}^{2}}{\rho}+P+\frac{B_{t}^{2}}{8 \pi}\right]=0,} \\
{\left[w+\frac{j_{n}^{2}}{2 \rho^{2}}+\frac{u_{t}^{2}}{2}+\frac{B_{t}^{2}}{4 \pi \rho}-\frac{B_{n}}{4 \pi j_{n}} \mathbf{B}_{\mathbf{t}} \mathbf{u}_{\mathbf{t}}\right]=0 .}
\end{gathered}
$$

Here $\mathbf{U}=\left(u_{n}, \mathbf{u}_{\mathbf{t}}\right)$ is the bulk velocity, $w=\varepsilon+P / \rho$ is the gas enthalpy, $\varepsilon, P, \rho$ are the internal gas energy, pressure and density respectively. The subscripts $n$ and $t$ are used for the normal and transverse components respectively. We used the standard notations $[A]=$ $A_{2}-A_{1}$ for the jump of a function $A$ between the downstream and upstream regions. In the MHD case the relations equivalent to (1)-(4) were obtained by de Hoffmann and Teller (1950). The equations are valid in the case of a magnetic field frozen-into moving plasma, where $\mathbf{E}=-\frac{\mathbf{U}}{c} \times \mathbf{B}$. A specific feature of MHD shock waves is the so-called coplanarity theorem (e.g., Landau and Lifshitz 1984) saying that the upstream and downstream magnetic 
Fig. 1 A sketch illustrating the coplanarity theorem for a plane ideal MHD-shock. The upstream and downstream bulk velocities $\mathbf{U}_{\mathbf{1}}$ and $\mathbf{U}_{2}$, magnetic fields $\mathbf{B}_{1}$ and $\mathbf{B}_{\mathbf{2}}$ and the shock normal $\mathbf{N}$ all lie in the same plane. The shock is at rest in the reference frame where also $u_{t 1}=0$. The shock is of infinitesimal width in the sketch. Simulated structure of the transition region of a collisionless shock is shown in Fig. 2 and Fig. 3 where its finite width is apparent

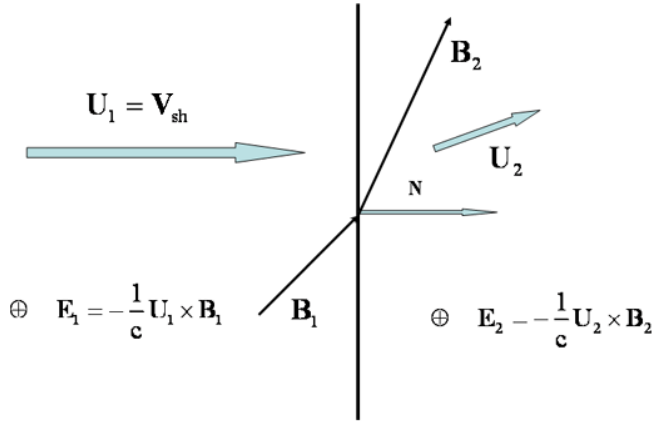

MHD-shock geometry (the coplanarity theorem)

fields $\mathbf{B}_{1}$ and $\mathbf{B}_{2}$ and the shock normal all lie in the same plane as it is illustrated in Fig. 1. It is important to note that if $B_{n} \neq 0$ there is an especial reference frame where local velocity $\mathbf{U}$ and magnetic field $\mathbf{B}$ are parallel both in the upstream and downstream, providing $\mathbf{E}=0$.

From (1)-(4) one may obtain a generalised Rankine-Hugoniot (RH) adiabat

$$
\left.\varepsilon_{2}-\varepsilon_{1}+\frac{1}{2}\left(\frac{1}{\rho_{2}}-\frac{1}{\rho_{1}}\right)\left\{\left(P_{2}+P_{1}\right)+\frac{1}{8 \pi}\left(B_{t 2}-B_{t 1}\right)^{2}\right)\right\}=0 .
$$

The RH adiabat connects the macroscopic parameters downstream of the flow once the upstream state is known. In a parallel shock $\left(\mathbf{B}_{t}=0\right)$

$$
\begin{aligned}
r & =\frac{\rho_{2}}{\rho_{1}}=\frac{\left(\gamma_{\mathrm{g}}+1\right) \mathcal{M}_{\mathrm{s}}^{2}}{\left(\gamma_{\mathrm{g}}-1\right) \mathcal{M}_{\mathrm{s}}^{2}+2}, \\
\frac{T_{2}}{T_{1}} & =\frac{\left[2 \gamma_{\mathrm{g}} \mathcal{M}_{\mathrm{s}}^{2}-\left(\gamma_{\mathrm{g}}-1\right)\right]\left[\left(\gamma_{\mathrm{g}}-1\right) \mathcal{M}_{\mathrm{s}}^{2}+2\right]}{\left(\gamma_{\mathrm{g}}+1\right)^{2} \mathcal{M}_{\mathrm{s}}^{2}},
\end{aligned}
$$

where $\gamma_{\mathrm{g}}$ is the gas adiabatic exponent. We restrict ourselves here to a fast mode shock where $c_{\mathrm{s} 1}<u_{1}$, and $v_{\mathrm{a} 2}<u_{2}<c_{\mathrm{s} 2}$, for $v_{\mathrm{a} 1}<c_{\mathrm{s} 1}$. The phase velocity $v_{\mathrm{a} 2}$ is the Alfvén velocity in the downstream, $c_{\mathrm{s} 1}, c_{\mathrm{s} 2}$ are the sound speeds in the upstream and downstream respectively. We define here the shock Mach numbers as $\mathcal{M}_{\mathrm{s}}=v_{\mathrm{sh}} / c_{\mathrm{s} 1}$ and $\mathcal{M}_{\mathrm{a}}=v_{\mathrm{sh}} / v_{\mathrm{a} 1}$.

In the case of a perpendicular shock $\left(B_{n}=0\right)$ the compression ratio is

$$
\begin{aligned}
r & =\frac{\rho_{2}}{\rho_{1}}=\frac{2\left(\gamma_{\mathrm{g}}+1\right)}{\psi+\left(\psi^{2}+4\left(\gamma_{\mathrm{g}}+1\right)\left(2-\gamma_{\mathrm{g}}\right) \mathcal{M}_{\mathrm{a}}^{-2}\right)^{1 / 2}}, \\
\psi & =\left(\gamma_{\mathrm{g}}-1\right)+\left(2 \mathcal{M}_{\mathrm{s}}^{-2}+\gamma_{\mathrm{g}} \mathcal{M}_{\mathrm{a}}^{-2}\right) .
\end{aligned}
$$

In a single fluid strong shock with $\mathcal{M}_{\mathrm{s}} \gg 1$ and $\mathcal{M}_{\mathrm{a}} \gg 1$ one gets

$$
T_{2} \approx 2 \cdot \frac{\left(\gamma_{\mathrm{g}}-1\right)}{\left(\gamma_{\mathrm{g}}+1\right)^{2}} \mu v_{\mathrm{sh}}^{2}=1.38 \times 10^{7} v_{\mathrm{s} 8}^{2}(\mathrm{~K}),
$$

for any magnetic field inclination (e.g., Draine and McKee 1993). The mass per particle $\mu$ was assumed to be [1.4/2.3] $m_{\mathrm{H}}$ and $v_{\mathrm{s} 8}$ is the shock velocity in $10^{8} \mathrm{~cm} \mathrm{~s}^{-1}$.

The RH adiabat does not depend on the exact nature of the dissipation mechanisms that provide the transition between the states 1 and 2. It assumes a single-fluid motion in regular 
electromagnetic fields. However, the dissipative effects control the thickness of the shock transition layer. In the case of a weak shock of Mach number $\mathcal{M}_{\mathrm{s}}-1 \ll 1$ the thickness is large enough, allowing a macroscopic hydrodynamical description of the fluid inside the shock transition layer (e.g., Landau and Lifshitz 1984). The gas shock width $\Delta$ in collisional hydrodynamics without magnetic fields is given by

$$
\Delta=\frac{8 a V^{2}}{\left(P_{2}-P_{1}\right)\left(\partial^{2} V / \partial P^{2}\right)_{s}} .
$$

Following Landau and Lifshitz (1959) the gas shock width in (10) can be expressed through the viscosities $\eta$ and $\zeta$, and thermal conductivity $\kappa$, since

$$
a=\frac{1}{2 \rho v_{\mathrm{s}}^{3}}\left[\left(\frac{4}{3} \eta+\zeta\right)+\kappa\left(\frac{1}{c_{\mathrm{v}}}-\frac{1}{c_{\mathrm{p}}}\right)\right] .
$$

Here $c_{\mathrm{v}}$ and $c_{\mathrm{p}}$ are specific heats at constant volume and at constant pressure respectively. Extrapolating (10) to a shock of finite strength where $P_{2}-P_{1} \sim P_{2}$, one may show that the gas shock width $\Delta$ is of the order of the mean free path $\lambda$.

It is instructive to note that the entropy is non-monotonic inside the finite width of a weak gas shock $\left(\mathcal{M}_{\mathrm{s}}-1\right) \ll 1$ and the total RH jump of the entropy $\Delta s$ across the shock is of the third order in $\left(\mathcal{M}_{\mathrm{s}}-1\right)$ :

$$
\Delta s=\frac{1}{12 T_{1}}\left(\frac{\partial^{2} V}{\partial P^{2}}\right)_{s}\left(P_{2}-P_{1}\right)^{3} \propto\left(\mathcal{M}_{\mathrm{s}}-1\right)^{3},
$$

while the density, temperature and pressure jumps are $\propto\left(\mathcal{M}_{\mathrm{s}}-1\right)$ (Landau and Lifshitz 1959).

In plasma shocks the shock structure is more complex because of a relatively slow electron-ion temperature relaxation. Such a shock consists of an ion viscous jump and an electron-ion thermal relaxation zone. In the case of plasma shocks the structure of the ion viscous jump is similar to the single fluid shock width structure discussed above and can be studied accounting for the entropy of an isothermal electron fluid. The shock ion viscous jump has a width of the order of the ion mean free path. The scattering length (the mean free path to $\pi / 2$ deflection) $\lambda_{\mathrm{p}}$ of a proton of velocity $v_{7}$ (measured in $100 \mathrm{~km} \mathrm{~s}^{-1}$ ) due to binary Coulomb collisions with plasma protons of density $n$ (measured in $\mathrm{cm}^{-3}$ ) can be estimated as $\lambda_{\mathrm{p}} \approx 7 \times 10^{14} v_{7}^{4} n^{-1} \mathrm{~cm}$ (Spitzer 1962). After the reionisation $(z<6)$ the Coulomb mean free path in the WHIM of overdensity $\delta$ is $\lambda_{\mathrm{p}} \approx 3.5 \times 10^{21} v_{7}^{4} \delta^{-1}(1+z)^{-3}\left(\Omega_{\mathrm{b}} h^{2} / 0.02\right)^{-1} \mathrm{~cm}$. Here and below $\Omega_{\mathrm{b}}$ is the baryon density parameter. The mean free path due to Coulomb collisions is typically some orders of magnitude smaller than that for the charge-exchange collisions in the WHIM after reionisation. The ion-electron thermal relaxation occurs on scales about $\lambda_{\mathrm{e}} \times \sqrt{m_{\mathrm{p}} / Z m_{\mathrm{e}}}$. Since $\lambda_{\mathrm{e}} \sim \lambda_{\mathrm{p}}$, the width of the relaxation zone is substantially larger than the scale size of the ion viscous jump. The application of the single fluid shock model (1)-(4) to electron-ion plasmas assumes full ion-electron temperature relaxation over the shock width. For a discussion of the relaxation processes see e.g., Bykov et al. (2008-Chap. 8, this issue and references therein).

In a rarefied hot cosmic plasma the Coulomb collisions are not sufficient to provide the viscous dissipation of the incoming flow, and collective effects due to the plasma flow instabilities play a major role, providing the collisionless shocks, as it is directly observed in the heliosphere. The observed structure of supernova remnants (e.g., Weisskopf and Hughes 2006) is consistent with that expected if their forward shocks are collisionless. Moreover, the 
non-thermal synchrotron emission seen in radio and X-rays is rather a strong argument for high energy particle acceleration by the shock that definitely favours its collisionless nature. That allows us to suggest that cosmological shocks in a rarefied highly ionised plasma (after the reionisation epoch) are likely to be collisionless. There are yet very few observational studies of cosmological shocks (e.g., Markevitch and Vikhlinin 2007). We review some basic principles of collisionless shock physics in the next section.

\section{Collisionless Shocks}

Since the discovery of the solar wind in the early 1960's it has been realised that the rapid rise time of magnetic storms observed in the Earth suggested very thin collisionless shocks created by solar flares (see for a discussion Sagdeev 1966; Kennel et al. 1985). The thickness of a viscous jump in a strong collisional shock is of the order of a mean free path (see e.g., Zel'dovich and Raizer 1967). The Coulomb collision mean free path in the tenuous solar wind plasma is comparable to the Sun-Earth distance, and thus the magnetic storm rising time due to standard collisional shocks would exceed the observed time by orders of magnitude.

There are very specific features of collisionless plasma shocks (Sagdeev 1966). Shocks in dense enough plasma with frequent Coulomb collisions evolve very fast to Maxwellian particle distributions with very few particles at high energies. On the contrary, in collisionless plasma shocks, a small minority of particles could gain a disproportionate share of the energy and become non-Maxwellian. Collisionless shocks enable acceleration of a small fraction of the particles to very high energies. Moreover, the accelerated particles could carry away a substantial amount of the kinetic energy of the plasma flow dissipated at the shock. The energetic particles can penetrate far into the shock upstream gas, to create an extended shock precursor. The cold gas in the shock upstream is decelerated and pre-heated by the energetic particle and MHD-wave pressure on a scale larger than a mean free path of an energetic particle. This occurs not only at the bow shock of the Earth at moderately low energies, but also in astrophysical shocks at highly relativistic energies (e.g., Russell 2005).

A direct study of collisionless shock waves in a laboratory is an extremely difficult task. Most of the experimental data on collisionless shock physics are coming from space experiments. There are direct observational data on the shock wave structure in the interplanetary medium with clear evidence for ion and electron acceleration by the shocks (e.g., Tsurutani and Lin 1985; Russell 2005).

Computer simulations of the full structure of collisionless shock waves describe the kinetics of multi-species particle flows and magneto-hydrodynamic (MHD) waves in the strongly-coupled system. The problem is multi-scale. It requires a simultaneous treatment of both "microscopic" structure of the subshock at the thermal ion gyroradii scale where the injection process is thought to occur, and an extended "macroscopic" shock precursor due to energetic particles. The precursor scale is typically more than $10^{9}$ times the microscopic scale of the subshock transition region.

Energetic particles could be an essential component in the WHIM and clusters of galaxies. Nonthermal particle acceleration at shocks is expected to be an efficient process at different evolutional stages of clusters. Being the governing process of the supernova remnant collisionless shock formation, nonlinear wave-particle interactions are responsible for both shock heating and compression of the thermal gas, as well as for creation of an energetic particle population. 


\subsection{Micro Processes in Collisionless Shocks}

In the strong enough collisionless shocks (typically with a Mach number above a few) resistivity cannot provide energy dissipation fast enough to create a standard shock transition (e.g., Kennel et al. 1985) on a microscopic scale. Ion instabilities are important in such shocks that are called supercritical.

At the microscopic scale the front of a supercritical shock wave is a transition region occupied by magnetic field fluctuations of an amplitude $\delta B / B \sim 1$ and characteristic frequencies of about the ion gyro-frequency. Generation of the fluctuations is due to instabilities in the interpenetrating multi-flow ion movements. The width of the transition region of a quasi-parallel shock wave reaches a few hundred ion inertial lengths defined as $l_{i}=c / \omega_{\mathrm{pi}} \approx 2.3 \times 10^{7} n^{-0.5} \mathrm{~cm}$. Here $\omega_{\mathrm{pi}}$ is the ion plasma frequency and $n$ is the ionised ambient gas number density measured in $\mathrm{cm}^{-3}$. The ion inertial length in the WHIM can be estimated as $l_{i} \approx 5.1 \times 10^{10} \delta^{-1 / 2}(1+z)^{-3 / 2}\left(\Omega_{\mathrm{b}} h^{2} / 0.02\right)^{-1 / 2} \mathrm{~cm}$, providing the width of the collisionless shock transition region is smaller by many orders of magnitude than the Coulomb mean free path (that is in the kiloparsec range).

The transition region of a quasi-perpendicular shock is somewhat narrower. The wave generation effects at the microscopic scale have been studied in some detail with hybrid code simulations (e.g., Quest 1988). The large-amplitude magnetic field fluctuations in the shock transition region were directly measured in the interplanetary medium (see e.g., Kan et al. 1991).

There are a few ways to simulate numerically the kinetics of the collisionless plasma phenomena. The most comprehensive study of the collisionless shock structure can be performed with the particle-in-cell (PIC) method where all the plasma components are considered as discrete particles in self-consistent fields. The PIC method allows one to resolve electron scale lengths and frequencies, but on the other hand it requires considerable computer resource. A serious constraint on PIC and other plasma particle simulations of collisionless shocks is that they must be done fully in three spatial dimensions (3D). Jones et al. (1998) have proved that PIC simulations with one or more ignorable dimensions artificially confine particles to field lines and particularly eliminate cross-field diffusion. The effect is especially important for simulations of a creation of a superthermal particle population. All three box dimensions must be involved in these simulations. Exact modelling of electron kinetics in collisionless shocks require PIC simulations (e.g., Hoshino and Shimada 2002; Schmitz et al. 2002). On the other hand, the bulk of the energy of a collisionless shock is carried by the ions and velocity relaxation processes are typically longer than the ion gyroperiods. Thus, though the basic shock physics evolve on ion spatial and temporal scales, the electron kinetic description requires fine resolution at electron scales.

A fairly good description of low-frequency processes of the ion dynamics in the shock transition layer can be achieved with hybrid codes (e.g., Winske and Omidi 1996 and references therein). Hybrid code modelling, which interprets protons as particles and electrons as an inertialess liquid, has made it possible to describe some important features of the (sub)shock waves at the microscopic scale of some hundred times the ion inertial length (e.g., Quest 1988; Lembege et al. 2004; Burgess et al. 2005).

A typical initialisation of a shock in the hybrid code simulations is to inject a relatively cold ion beam (say at the right-hand boundary) and to put a particle reflecting wall at the left-hand boundary of a simulation box. In that case the shock is moving, and the available simulation time is limited, given the finite size of the box. The limited simulation time and the particle statistics per cell are challenging the direct modelling of the origin and evolution of the energetic non-thermal particle population in a shock. To increase the statistics the macro-particle splitting method is used (see e.g., Quest 1992; Giacalone and Ellison 2000). 

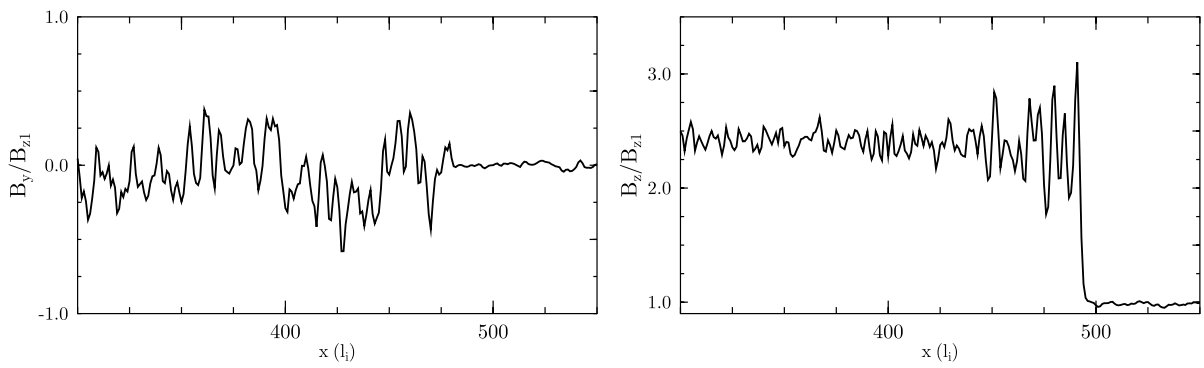

Fig. 2 Hybrid simulated magnetic fields of a quasi-perpendicular shock ( $80^{\circ}$ inclination). The shock propagates along the $x$-axis, while the initial regular magnetic field is in the $x-z$ plane. We show the $B_{y}$ and $B_{z}$ dependence on $x$ in the left and right panels respectively

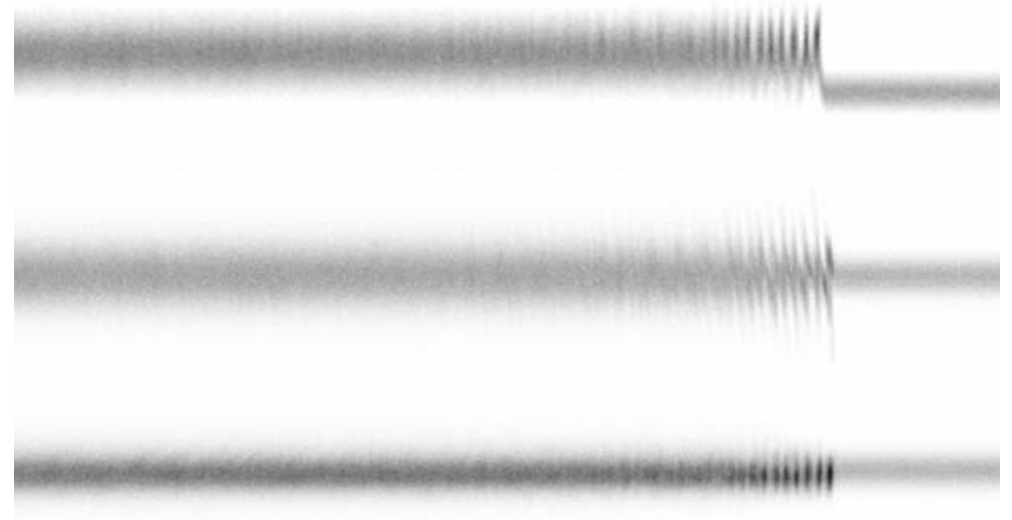

Fig. 3 Hybrid simulated proton phase density in a quasi-perpendicular shock ( $80^{\circ}$ inclination). The shock is moving from left to right in the reference frame where the particle reflecting wall is at rest. The figures show the proton phase densities in $v_{x}-x, v_{y}-x$ and $v_{z}-x$ projections from top to bottom respectively

In Fig. 2 we show the structure of the magnetic field in a quasi-perpendicular shock (inclination angle $\theta_{B n} \approx 80^{\circ}$ ) simulated with a hybrid code for the upstream plasma parameter $\beta \sim 1$. The parameter $\beta=\mathcal{M}_{\mathrm{a}}^{2} / \mathcal{M}_{\mathrm{s}}^{2}$, characterises the ratio of the thermal and magnetic pressures. The shock is propagating along the $x$-axis from the left to the right. The magnetic field is in the $x-z$ plane. The system is periodic in the $y$-dimension. Phase densities of protons $v_{x}-x, v_{y}-x, v_{z}-x$ are shown in Fig. 3 in the reference frame where the particle reflecting wall (at far left) is at rest while the shock front is moving. The incoming plasma beam in the simulation was composed of protons (90\%), alpha particles $(9.9 \%)$ and a dynamically insignificant fraction of oxygen ions (O VII).

In most of the cases non-relativistic shocks simulated with different hybrid codes had the upstream plasma parameter $\beta \sim 1$. In some cosmological shocks, for example in hot X-ray clusters, plasma parameter $\beta$ could be $\sim 100$ (see Bykov et al. 2008-Chap. 8, this issue). The nature of collisionless shocks in the hot low magnetised plasmas could be different from that in case of $\beta \sim 1$. While the processes of shock formation in the high beta plasmas still require a careful study, there are two experimental studies establishing the existence of the 
collisionless shocks for that case. A collisionless shock in a laboratory experiment with a laser-produced ablating plasma of $\beta \sim 300$ was found to have a thickness about $10 \mathrm{c} / \omega_{\text {pe }}$, that is orders of magnitude less than the Coulomb mean free path of both ions and electrons in that system (see Bell et al. 1988). In space plasma the terrestrial bow shock under high beta conditions was observed with the ISEE 1 and ISEE 2 spacecraft by Farris et al. (1992). These measurements were compared with and found to be in agreement with the predicted values of the Rankine-Hugoniot relations using the simple adiabatic approximation and a ratio of specific heats, gamma, of 5/3. Large magnetic field and density fluctuations were observed, but average downstream plasma conditions well away from the shock were relatively steady, near the predicted Rankine-Hugoniot values. The magnetic disturbances persisted well downstream and a hot, dense ion beam was detected leaking from the downstream region of the shock.

\subsection{Heating of Ions in Collisionless Shocks}

The heating processes in collisionless shocks are non-trivial. The irreversible transformation of a part of the kinetic energy of the ordered bulk motion of the upstream flow into the energy of the random motions of plasma particles in the downstream flow in collisional shocks is due to Coulomb or atomic particle collisions. In collisional non-radiative shocks without slowly relaxing molecular components, the standard single-fluid RH relations are applicable just after a few collisional lengths. The standard single-component shock model predicts a particle temperature $\mathrm{k} T=(3 / 16) m v_{\mathrm{sh}}^{2}$ for $\gamma=5 / 3$.

The particle distributions in the collisionless shocks are not Maxwellian. Thus, instead of the standard equilibrium temperature the appropriate moments of the particle distribution function characterising the width of randomised velocity distributions are used. Moreover, the particle velocity distributions are typically anisotropic. It is clearly seen in hybrid simulated proton phase density: in Fig. 3 the velocity distribution widths are different for different projections. One can see in Fig. 3 that a fraction of the incoming ions is reflected by the shock magnetic field jump providing multiple inter-penetrating flows of gyrating ions. Then the field fluctuations randomise the ion phases producing a "coarse-grained" distribution characterised by an effective temperature estimated as the second moment of the velocity distribution.

An analysis of interplanetary collisionless shock observations made with Advanced Composition Explorer by Korreck et al. (2007) indicated that quasi-perpendicular shocks are heating ions more efficiently than quasi-parallel shocks. It was also found that effective temperatures of different ions are not necessarily proportional to ion mass, but also depend on the shock inclination angle and plasma parameter $\beta$. The widths of the collisionless shocks are extremely narrow (below the astronomical unit) and thus, the observed temperatures would depend on the temperature equilibration processes (both Coulomb and collective) that we will discuss elsewhere (e.g., Bykov et al. 2008 - Chap. 8, this issue). The temperature equilibration of different plasma constituents in the WHIM can be studied with spatially resolved spectroscopic observations and thus is a good test for shock models.

\subsection{Heating of Electrons in Collisionless Shocks}

Electron kinetics in collisionless shocks are different from those of ions. Since most of the observable emission comes from the electrons, they require a careful study. Shocks transfer a fraction of the bulk kinetic energy of the ion flow into large amplitude nonlinear magnetic fluctuations on a short scale of the transition region (see Fig. 2). It is important that 


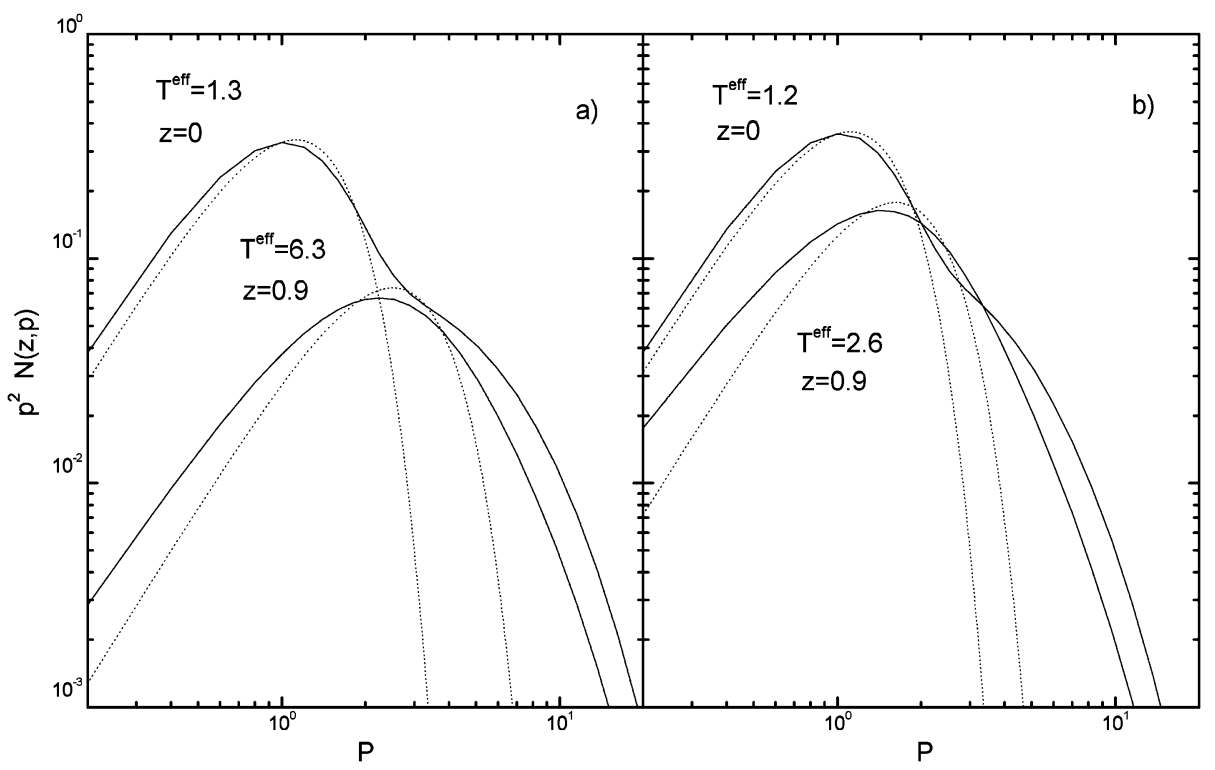

Fig. 4 Electron distribution function simulated in the model of electron heating by strong ion gyroradii scale magnetic fluctuations in a collisionless shock by Bykov and Uvarov (1999). The left panel is for $\alpha=\left\langle(\delta B / B)^{2}\right\rangle=0.4$, the right panel for $\alpha=0.1$

the thermal electron velocities in the ambient medium are higher than the shock speed for a shock Mach number $\mathcal{M}_{\mathrm{s}}<\sqrt{m_{\mathrm{p}} / m_{\mathrm{e}}}$, allowing for a nearly-isotropic angular distribution of the electrons. Non-resonant interactions of these electrons with the large-amplitude turbulent fluctuations in the shock transition region could result in collisionless heating and pre-acceleration of the electrons (Bykov and Uvarov 1999). In Fig. 4 a simulated electron distribution $\left(p^{2} N(z, p)\right)$ is shown as a function of the dimensionless electron momentum $p / \sqrt{2 m_{\mathrm{e}} T_{1}}$, where $T_{1}$ is the initial electron temperature in the far upstream $(z \rightarrow-\infty)$. The solid curves are the simulated electron distribution functions at the left boundary $(\tilde{z}=0)$ of the transition region clearly seen in Fig. 2, and at the end of the region $(\tilde{z}=1)$. Dotted lines are the Maxwellian distribution fits allowing to estimate the effective electron temperatures $T^{\text {eff }}$ measured relative to $T_{1}$. Note that $T^{\mathrm{eff}}=1.2$ at $\tilde{z}=0$ because of the electron diffusivity effect. One may also clearly see the appearance of non-thermal tails indicating a Fermi type acceleration. It is worth noting that the presence of large-amplitude waves in the shock transition region erodes many of the differences between quasi-parallel and perpendicular shocks, making the electron injection mechanism in that model to be similar for these shocks.

The analysis of observational data on both interplanetary and supernova shocks by Ghavamian et al. (2007) indicates that the electron heating efficiency i.e. $T_{\mathrm{e}} / T_{\mathrm{i}}$ is a declining function of the shock velocity. These authors discussed a model of electron heating with a constant level of electron heating over a wide range of shock speeds (see also Fig. 4 in Bykov and Uvarov 1999), while the ion heating is an increasing function of shock velocity.

\subsection{Gas Heating and Entropy Production in Weak Internal Shocks}

Heating/acceleration efficiency with weak and moderate strength MHD shocks can be estimated by calculating the energy dissipation rate $\dot{\varepsilon}_{\mathrm{h}}$ of a directed gas motion per unit area of 
a weak shock. Defining $\dot{\varepsilon}_{\mathrm{h}}=v_{\mathrm{sh}} \rho T \Delta s$, where $\Delta s$ is the difference of the entropies (per unit mass) behind and ahead of the shock front, one can evaluate the thermal gas heating as

$$
\dot{\varepsilon}_{\mathrm{h}}=(5 / 4)\left(\mathcal{M}_{\mathrm{s}}-1\right)^{3} v_{\mathrm{sh}} \varepsilon_{\mathrm{T}},
$$

where $\varepsilon_{T}$ is the gas internal energy per unit volume (cf. (11)). The energy transfer to the reflected nonthermal particles can be estimated from:

$$
\dot{\varepsilon}_{\mathrm{CR}}=\left(\mathcal{M}_{\mathrm{s}}-1\right)^{2} v_{\mathrm{sh}} \varepsilon_{\mathrm{B}},
$$

where $\varepsilon_{B}$ is the magnetic energy density (Bykov and Toptygin 1987). From (12) and (13) one can see that the gas heating is of third order in $\left(\mathcal{M}_{\mathrm{s}}-1\right) \ll 1$ (cf. Landau and Lifshitz 1959), while the wave damping due to the particle acceleration is of second order. Note that in the outer parts of galaxy clusters one would typically expect $\varepsilon_{\mathrm{T}} \gg \varepsilon_{\mathrm{B}}$. However, the central regions of such a cluster could have $\varepsilon_{\mathrm{T}}$ comparable to $\varepsilon_{\mathrm{B}}$, as it is the case in the Milky Way. Thus, the weak shocks in the central regions could efficiently accelerate nonthermal particles, reducing the heating of the gas. Particle acceleration by an ensemble of large scale shocks in a cluster of galaxies can create a population of non-thermal particles of sizeable pressure. This may imply a non-steady evolution of non-thermal pressure as modelled by Bykov (2001).

\section{Energetic Particle Acceleration in Collisionless Shocks}

The reflected ions with a gyro-radius exceeding the width of the shock transition region can then be efficiently accelerated, via the Fermi mechanism, by converging plasma flows carrying magnetic inhomogeneities and MHD waves. In perpendicular shock a net transverse particle momentum gain is due to the work of the electric field on the particle drift motion. The electric field perpendicular to the shock normal exists in all the reference frames for the perpendicular shock. The particle of a momentum $\mathbf{p}$ crossing back and forth the shock front and being scattered by MHD waves carried with a flow of velocity $\mathbf{u}$ would undergo a momentum increment $\Delta p \approx \mathbf{p} \cdot \frac{\mathbf{u}}{v}+O\left((u / v)^{2}\right)$ per scattering. A velocity profile in the plane shock is illustrated by the dashed line in Fig. 5 in the test particle case where one neglects the back reaction effect of accelerated particles on the shock. One way to calculate the

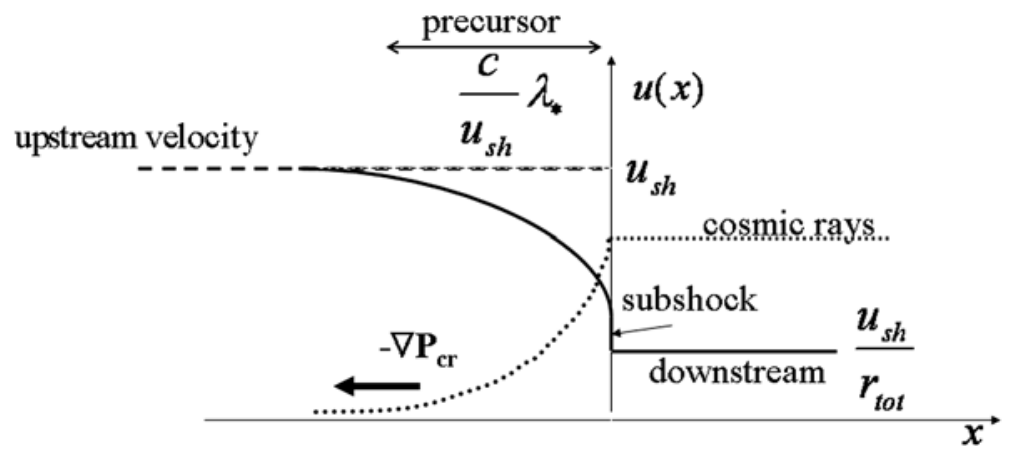

Fig. 5 A sketch illustrating the structure of a cosmic-ray modified shock. The dashed line is the shock velocity jump corresponding to the test particle case. The dotted line is a spatial distribution of accelerated particles at some momentum $p \gg p_{0}$. The solid line is the CR modified shock velocity profile with the precursor and subshock indicated 
accelerated particle spectra in a scattering medium is to use the kinetic equation in the diffusion approximation. The scattering medium in that approach is characterised by momentum dependent particle diffusion coefficients $k_{1}(p)$ and $k_{2}(p)$. The shock is considered as a bulk velocity jump (see the dashed line in Fig. 5) assuming that the test particles are injected at $p=p_{0}$ and the gyroradii of the particles are larger than the shock width. Therefore, in a test particle case particles must be injected at some super-thermal energy to be accelerated by the shock. A solution to the kinetic equation for a nearly isotropic test particle distribution in the phase space is a power-law momentum distribution $f(p, x) \propto\left(p / p_{0}\right)^{-b}, p \geq p_{0}$ where the index

$$
b=\frac{3 r}{r-1}
$$

depends on the shock compression ratio $r$ (Axford et al. 1977; Krymskii 1977; Bell 1978; Blandford and Ostriker 1978). The CR spatial distribution in the model is illustrated in Fig. 5. For a strong shock of $\mathcal{M}_{\mathrm{s}} \gg 1$ and $\mathcal{M}_{\mathrm{a}} \gg 1$ the compression ratio given by (6) and (8) is close to 4 if $\gamma_{\mathrm{g}}=5 / 3$ (or even larger if relativistic gas dominates the equation of state). The pressure of the accelerated particles is

$$
P_{\mathrm{CR}}=\frac{4 \pi}{3} \int_{p_{0}}^{\infty} p v f(p, x) p^{2} \mathrm{~d} p .
$$

Then for $b=4$ one may see that $P_{\mathrm{CR}} \propto \ln \left(p_{\max } / p_{0}\right)$ indicating a potentially large cosmic ray (CR) pressure, if CRs are accelerated to $p_{\max } \gg p_{0}$. The maximal energy of accelerated test particles depends on the diffusion coefficients, bulk velocity and scale-size of the system. The finite scale-size of the shock is usually accounted for by an energy dependent free escape boundary located either in the upstream or in the downstream. For electrons $p_{\max }$ can also be limited by synchrotron (or inverse-Compton) losses of relativistic particles.

The test particle shock acceleration time $\tau_{\mathrm{a}}(p)$ can be estimated from the equation

$$
\tau_{\mathrm{a}}(p)=\frac{3}{u_{1 n}-u_{2 n}} \int_{p_{0}}^{p}\left(\frac{k_{1}(p)}{u_{1 n}}+\frac{k_{2}(p)}{u_{2 n}}\right) \frac{\mathrm{d} p}{p}
$$

where the normal components of the shock upstream and downstream bulk velocities $u_{1 n}, u_{2 n}$ are measured in the shock rest frame. Estimations based on a more rigorous approach distinguishing between the mean acceleration time and the variance does not change the results substantially, given the uncertainties in the diffusion model.

\section{Cosmic-Ray Modified Multifluid Shocks}

The efficiency of the upstream plasma flow energy conversion into nonthermal particles could be high enough providing a hard spectrum of nonthermal particles up to some maximal energy $\varepsilon_{\star}$. If the efficiency of ram energy transfer to the energetic particles is high enough, an extended shock precursor appears due to the incoming plasma flow deceleration by the fast particle pressure. The precursor scale $L$ is of the order of $\left(c / v_{\mathrm{sh}}\right) \lambda_{\star}$-orders of magnitude larger than the width of the shock transition region (see Fig. 5). Here $\lambda_{\star}$ is the maximal mean free path of a particle in the energy-containing part of the spectrum and $v_{\mathrm{sh}}$ is the shock velocity. We shall later refer to these energetic particles as cosmic rays.

It has been shown that the front of a strong collisionless shock wave consists of an extended precursor and a viscous velocity discontinuity (subshock) of a local Mach number 
that is smaller than the total Mach number of the shock wave (see Fig. 5). The compression of matter at the subshock can be much lower than the total compression of the medium in the shock wave with allowance for high compression in the precursor. We shall refer later to such shocks as CR-modified.

The large scale ("macroscopic") structure of a CR-modified shock can be modelled by a two-fluid approach with a kinetic description of nonthermal particles (see e.g., Blandford and Eichler 1987; Berezhko et al. 1996; Malkov and Drury 2001; Blasi 2004 and references therein) or by a Monte Carlo method (e.g., Jones and Ellison 1991; Ellison et al. 1996). In both methods some suitable parameterisation of particle scattering process must be postulated a priori. Monte-Carlo simulations, however, have no assumption of isotropy for particle distributions, and that allows an internally self-consistent treatment of thermal particle injection. While the injection depends on the assumptions made for the particle pitch-angle scattering, these assumptions are applied equally to particles of all energies. The Monte Carlo technique eliminates a free injection parameter, which is present in all models based on the diffusion approximation and is used to set the injection efficiency. The strong feedback between injection, shock structure, and magnetic field amplification makes this property of the Monte Carlo technique particularly important. The Monte Carlo technique allows to iteratively obtain a shock velocity profile and particle distribution function conserving mass, momentum and energy fluxes taking into account the nonlinear feedback from the accelerated energetic particles.

In Fig. 6 Monte Carlo simulated proton spectra (multiplied by $\left.\left[p /\left(m_{\mathrm{p}} c\right)\right]^{4}\right)$ are shown, in the downstream shock from Vladimirov et al. (2006). To illustrate the dependence of the maximal energy of an accelerated proton on the system scale size, a free escape boundary condition was applied at some distance from the subshock position in the shock rest frame. The heavy solid and dotted curves in the right panel correspond to the free escape boundary located at a distance $10^{4} r_{\mathrm{g} 1}$ (where $r_{\mathrm{g} 1}=m_{\mathrm{p}} v_{\mathrm{sh}} \mathrm{c} / \mathrm{e} B_{1}$ ), the dashed curve has $10^{3} r_{\mathrm{g} 1}$, and the light solid curve has $10^{5} r_{\mathrm{g} 1}$. The simulations were done for a supernova shock in the interstellar medium with a shock speed $v_{\mathrm{sh}}=5000 \mathrm{~km} \mathrm{~s}^{-1}$ and an unshocked proton number
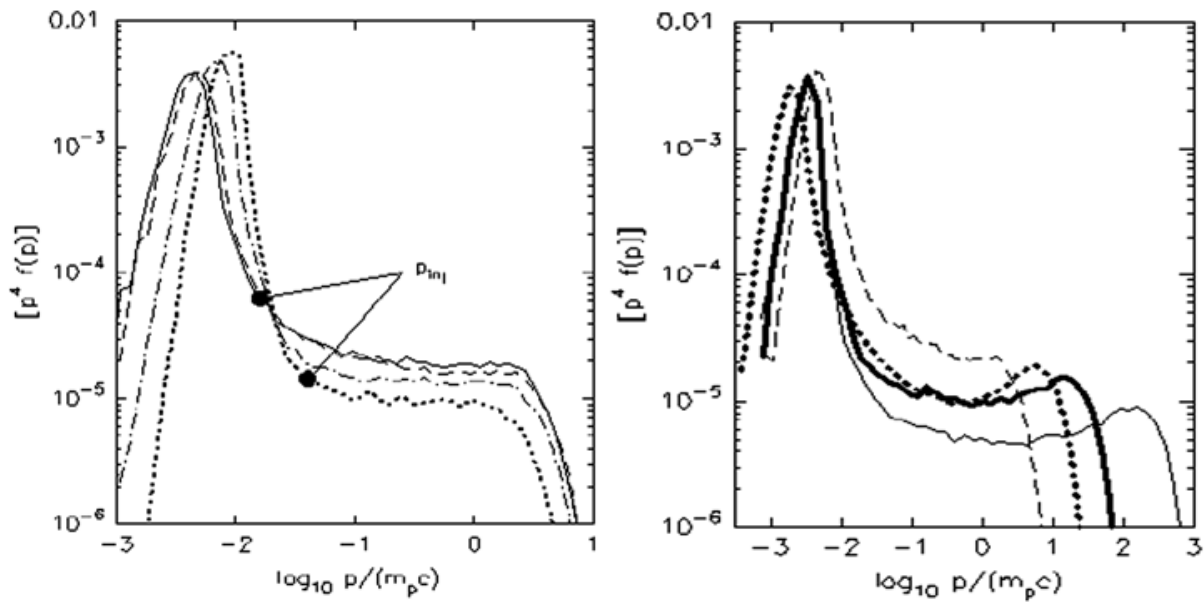

Fig. 6 Spectra of protons accelerated by a strong shock. The spectra were simulated with a non-linear Monte-Carlo model which accounts for particle injection and magnetic field amplification by the shock (for details see Vladimirov et al. 2006). On the left panel the transitions from the thermal-like component to the high energy tail are marked by $p_{\text {inj. }}$. On the right panel different curves correspond to different locations of the free escape boundary (see in the text) 
density $n_{1}=1 \mathrm{~cm}^{-3}$. In the left panel the spectra are given for the same position of the free escape boundary, but for different prescriptions of the scattering model.

\subsection{Magnetic Field Amplification in CR-Dominated Shocks}

An important predicted feature of strong shocks with efficient CR acceleration is the possibility to amplify an initial seed magnetic field by orders of magnitude (e.g., Bell and Lucek 2001; Bell 2004). CR current and CR pressure gradient upstream of the strong shock could drive magnetic fluctuations on the shock precursor scale length. The CR-shock precursor scale $L$ is $\sim\left(c / v_{\mathrm{sh}}\right) \lambda_{\star}$ which is expected to be above a kpc, moreover, the width is $L \gtrsim 100 \mathrm{kpc}$ for a shock of a size comparable to that of a galaxy cluster. The precursor scale size $L$ is $\gg 10^{9}$ times larger than the subshock transition region where strong small scale magnetic field fluctuations are directly produced by instabilities of super-Alfvénic bulk plasma flows illustrated in Fig. 2. That small scale fluctuations are responsible for bulk plasma motion dissipation process and adiabatic amplification of the transverse magnetic field in collisionless shocks. At the same time the collisionless dissipation process is thought to inject a minor fraction of incoming particles to be accelerated to high energies by Fermi mechanism. Recent models of diffusive shock acceleration allows a substantial fraction (say, 30\%) of the MHD shock ram pressure to be converted to accelerated particles filling a vicinity of the shock of the scale $L$. The large scale current and density gradient of the accelerated CRs may convert a fraction of the CR energy to magnetic field due to multifluid instabilities of different kinds providing a way to amplify the initial magnetic field by a factor larger than the shock compression ratio.

Recent non-linear simulations of magnetic field amplification in diffusive shock acceleration by a Monte-Carlo model (Vladimirov et al. 2006) and a kinetic model (Amato and Blasi 2006) confirmed the possibility of a significant effect. The amplitude of the fluctuating magnetic field energy density $\mathrm{W}_{\mathrm{B}}$ is of the order of the shock accelerated $\mathrm{CR}$ pressure which is in turn a substantial fraction of the shock ram pressure $0.5 \rho_{1} v_{\mathrm{sh}}^{2}$. Here $\rho_{1}$ is the shock upstream ambient gas density.

For typical cluster parameters the discussed mechanism could provide a $\mu \mathrm{G}$ range magnetic field amplitude in a hundred kpc range scale of CR-modified shock precursor. The Faraday rotation measure $R M$ provided by a strong CR-dominated shock in a cluster can reach values of $\gtrsim 10 \mathrm{rad} \mathrm{m}^{-2}$ and even a few times higher. For the case of the socalled Bohm diffusion model the rotation measure $R M$ is proportional to the maximal energy of the ions in the energy-containing part of the CR-spectrum accelerated by the shock. Radio observations, Faraday rotation and synchrotron-Compton emission measurements are used to estimate the magnetic fields in clusters (e.g., Carilli and Taylor 2002; Newman et al. 2002). Large filaments of polarised radio emission of scale size about $400 \mathrm{kpc}$ were discovered by Govoni et al. (2005) in the halo of the cluster of galaxies Abell 2255 and by Bagchi et al. (2006) in Abell 3376 (see Fig. 8). They could be connected to large scale shocks due to accretion/merging activity of the cluster.

\subsection{Gas Heating and Entropy Production in Strong CR-Modified Shocks}

An exact modelling of a collisionless shock structure taking into account the nonthermal particle acceleration effect requires the nonperturbative self-consistent description of a multicomponent and multi-scale system including strong MHD-turbulence dynamics. Such a modelling is not feasible at the moment. Instead, a simplified description of a multi-fluid strong shock structure can be used with an appropriate parameterisation of the extended 
Fig. 7 Total compression ratio $r_{\text {tot }}$ of a strong MHD shock modified by efficient particle acceleration as a function of the energy escape flux $Q_{\mathrm{esc}} / \Pi_{\mathrm{kin}}$ carried by energetic particles, where $\Pi_{\mathrm{kin}}=\rho_{1} v_{\mathrm{sh}}^{3} / 2$. The upper curve (dotted) corresponds to an effective adiabatic exponent $\gamma=4 / 3$ (relativistic gas), while the lower (solid) curve corresponds to $\gamma=5 / 3$

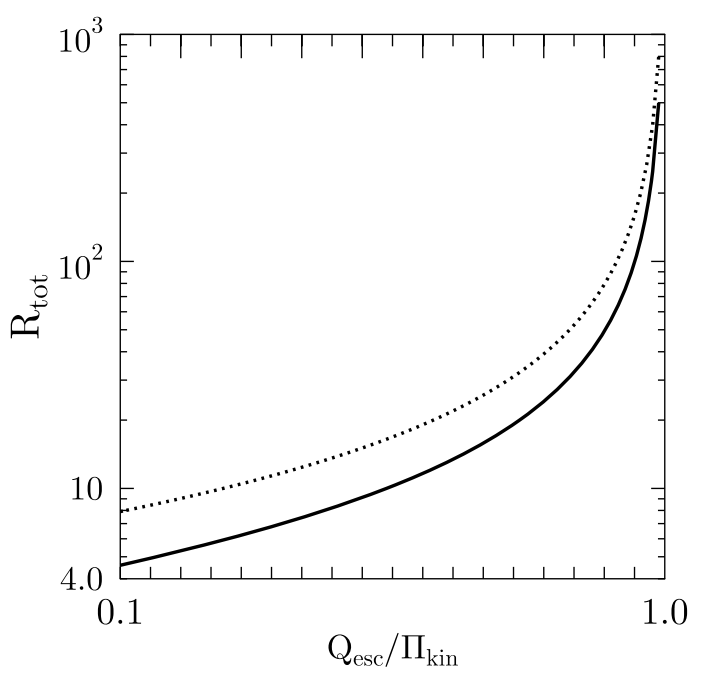

pre-shock and of the gas subshock. The predicted observable characteristics of the shocks can be confronted to the observational data. We will now discuss the effects of plasma heating by modified shocks and then make some specific predictions for possible observational tests.

In the shocks with efficient high energy particle acceleration the energy flux carried away by escaping energetic particles $Q_{\text {esc }}$ must be accounted for in the energy continuity equations. The energy loss results in a lower effective adiabatic index, but it allows to increase the total compression of the gas in the shock downstream.

The total compression ratio $r_{\text {tot }}$ of a strong MHD shock modified by an efficient nonthermal particle acceleration can be estimated as

$$
r_{\mathrm{tot}}=\frac{\gamma+1}{\gamma-\sqrt{1+2\left(\gamma^{2}-1\right) Q_{\mathrm{esc}} / \rho_{a} v_{\mathrm{sh}}^{3}}},
$$

assuming that the energy density in the shock upstream is dominated by the ram pressure and that the CR escape is through the cut-off momentum regime (e.g., Malkov and Drury 2001). Here $\gamma$ is the effective adiabatic exponent. In Fig. 7 we illustrate the dependence of the compression ratio on $Q_{\mathrm{esc}} / \rho_{a} v_{\mathrm{sh}}^{3}$ for $\gamma=4 / 3$ and $5 / 3$ assuming that the effective adiabatic exponent is between the two values depending on the spectrum of the accelerated relativistic particles.

The distribution function of nonthermal particles and the bulk flow profile in the shock upstream region are sensitive to the total compression ratio $r_{\text {tot }}$. Thus, the exact calculation of the escape flux $Q_{\text {esc }}$ can be performed only in fully nonlinear kinetic simulations. Nevertheless, an approximate iterative approach (e.g., in the Monte Carlo model discussed above) can be used to make the steady-state distribution function consistent with the shock compression assuming some diffusion model. The subshock is the standard gas viscous shock of a Mach number $\mathcal{M}_{\text {sub }}$. For that simplified two-fluid model of a strong CR-modified shock the effective ion temperature in the downstream $T_{\mathrm{i}}^{(2)}$ can be estimated for a shock of a given velocity, if $r_{\text {tot }}$ and $r_{\text {sub }}$ are known:

$$
T_{\mathrm{i}}^{(2)} \approx \phi\left(\mathcal{M}_{\mathrm{sub}}\right) \frac{\mu v_{\mathrm{sh}}^{2}}{\gamma_{\mathrm{g}} r_{\mathrm{tot}}^{2}\left(v_{\mathrm{sh}}\right)}, \quad \text { where } \quad \phi\left(\mathcal{M}_{\mathrm{sub}}\right)=\frac{2 \gamma_{\mathrm{g}} \mathcal{M}_{\mathrm{sub}}^{2}-\left(\gamma_{\mathrm{g}}-1\right)}{\left(\gamma_{\mathrm{g}}-1\right) \mathcal{M}_{\mathrm{sub}}^{2}+2} .
$$


Single fluid strong shock heating represents the limit $\mathcal{M}_{\text {sub }}=\mathcal{M}_{\mathrm{s}} \gg 1$, since there is no precursor in that case, resulting in (9). In single-fluid systems the compression ratio $r_{\text {tot }}=r_{\text {sub }} \rightarrow\left(\gamma_{\mathrm{g}}+1\right) /\left(\gamma_{\mathrm{g}}-1\right)$ does not depend on the shock velocity and (18) reduces to (9). However, in multi-fluid shocks the total compression ratio depends on the shock velocity and could be substantially higher than that in the single-fluid case. This implies somewhat lower postshock ion temperatures for the strong multi-fluid shock of the same velocity and could be tested observationally. It is convenient to introduce the scaling $r_{\text {tot }}\left(v_{\mathrm{sh}}\right) \propto v_{\mathrm{sh}}^{\xi}$ to describe the different cases of strong shock heating (see Bykov 2005 for details). Then from Eq. 18,

$T_{\mathrm{i} 2} \propto \phi\left(\mathcal{M}_{\text {sub }}\right) v_{\mathrm{sh}}^{2(1-\xi)}$. The subshock Mach number $\mathcal{M}_{\text {sub }}$ depends, in general, on $\mathcal{M}_{\mathrm{s}}$ and $\mathcal{M}_{\mathrm{a}}$. Thus, an index $\sigma$ approximates the velocity dependence of $\phi\left(\mathcal{M}_{\mathrm{sub}}\right) \propto v_{\mathrm{sh}}^{\sigma}$. Finally, if $T_{\mathrm{i} 2} \propto v_{\mathrm{sh}}^{a}$, then the index $a=2(1-\xi)+\sigma$. For the case of shock precursor heating by CR generated Alfvén waves, the index $a \approx 1.25$ (Bykov 2005).

A distinctive feature of multi-fluid shocks is their high gas compression $r_{\text {tot }}\left(v_{\mathrm{sh}}\right)$ that could be well above the single fluid shock limit $\left(\gamma_{\mathrm{g}}+1\right) /\left(\gamma_{\mathrm{g}}-1\right)$ (see Fig. 7). At the same time entropy production for a strong multi-fluid shock scales as $\left[r_{\mathrm{tot}}\left(v_{\mathrm{sh}}\right)\right]^{-\left(\gamma_{\mathrm{g}}+1\right)}$ and it is significantly reduced compared to the single-fluid shock of the same velocity. The effects are due to energetic particle acceleration and magnetic field amplification.

Energetic particles penetrate into the shock upstream region. They are coupled with the upstream gas through fluctuating magnetic fields (including the Alfvén waves generated by the energetic particles). Magnetic field dissipation provides gas preheating and entropy production in the extended shock precursor. Such a heated pre-shock region of $\mathrm{k} T \lesssim 0.5 \mathrm{keV}$ would appear as an extended filament of width $L \sim\left(c / v_{\mathrm{sh}}\right) \lambda_{\star} \gtrsim 3 \times 10^{14} \epsilon_{\star} B_{-6}^{-1} \mathrm{~cm}$. Here $\epsilon_{\star}$ (in $\mathrm{GeV}$ ) is the highest energy of the hard branch of the accelerated particle spectrum. If $B_{-6} \sim 0.1$ in the cluster outskirts and if the hard spectrum of energetic nuclei extends to $\sim 10^{9} \mathrm{GeV}$ (cf. Norman et al. 1995) we have $L \sim 1 \mathrm{Mpc}$ and even wider. Projected on a hot X-ray cluster, such filaments could produce a soft X-ray component "excessive" to that produced by the hot cluster. A warm gas $(\sim 0.2 \mathrm{keV})$ emission filament found with $X M M$-Network in the outskirts of the Coma cluster by Finoguenov et al. (2003) could be an extended heated precursor of a strong multi-fluid accretion shock. For a detailed review of the soft X-ray/EUV excesses see Durret et al. (2008-Chap. 4, this issue).

\subsection{ICM Entropy Production by Multifluid Accretion Shocks}

Cold gas falling into the dark matter (DM) dominated gravitational well passes through a strong accretion shock. The shock is a source of gas entropy production in the intercluster medium (ICM) (e.g., Knight and Ponman 1997; Tozzi and Norman 2001; Voit et al. 2003). The post-shock entropy $K=K_{\mathrm{b}} T / \rho^{2 / 3}$ used in the ICM analysis and simulations (e.g., Bialek et al. 2001) is related to the standard thermodynamic entropy $s$ through $K \propto \exp \left(s / c_{\mathrm{v}}\right)$. In the standard scenario with a single-fluid accretion shock the post-shock entropy scales $K_{\text {sf }} \propto v_{\text {sh }}^{2} \rho_{1}^{-2 / 3}$ (e.g., Voit et al. 2003).

The multi-fluid nature of the collisionless accretion shock modifies the standard scaling relation to be

$$
K_{\mathrm{mf}} \propto v_{\mathrm{sh}}^{2}\left[r_{\mathrm{tot}}\left(v_{\mathrm{sh}}\right)\right]^{-\left(1+\gamma_{\mathrm{g}}\right)} \phi\left(\mathcal{M}_{\mathrm{sub}}\right) \rho_{1}^{\left(1-\gamma_{\mathrm{g}}\right)} .
$$

The compression ratio in CR-shocks is higher than in a strong single-fluid shock of the same velocity resulting in reduced post-shock entropy production. For example, in the case of Alfvén heating the post-shock entropy of a multi-fluid shock reduces as $K_{\mathrm{mf}} / K_{\mathrm{sf}} \sim$ $\left(15 / \mathcal{M}_{\mathrm{a}}\right)$ for $\mathcal{M}_{\mathrm{a}}>15$ and $\mathcal{M}_{\mathrm{s}}^{2}>\mathcal{M}_{\mathrm{a}}$. Here and below in numerical estimations we assume $\gamma_{\mathrm{g}}=5 / 3$, though a non-thermal baryonic component could reduce the index $\gamma_{\mathrm{g}}$. 


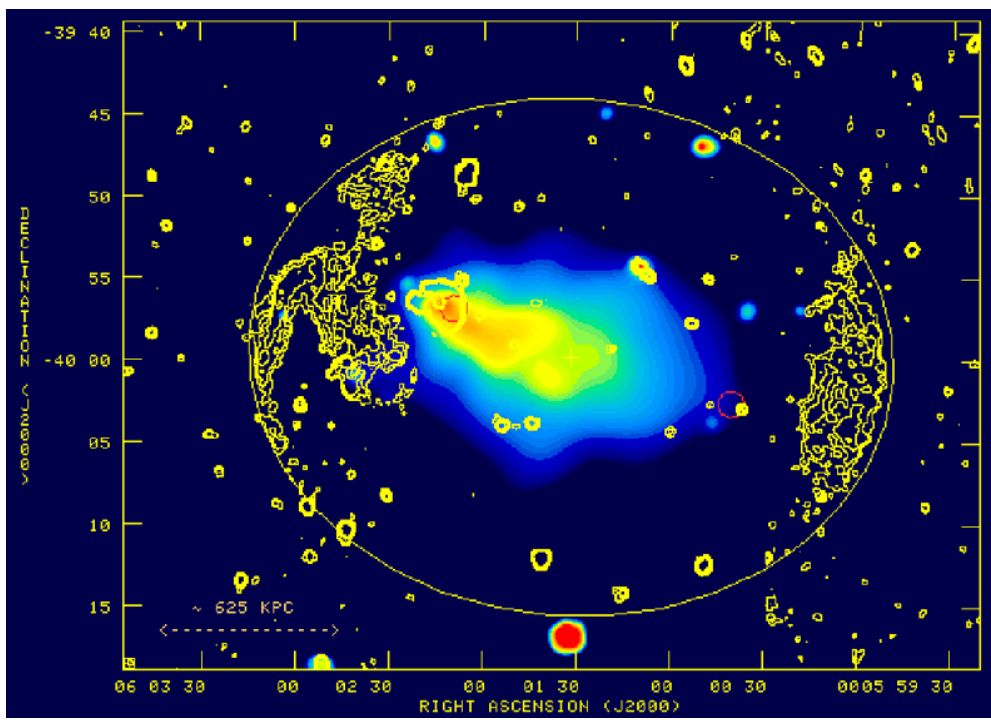

Fig. 8 Abell 3376 (taken from Bagchi et al. 2006: X-ray emission from XMM-Newton archive data, with VLA 1.4 GHz radio contours superimposed. The ellipse shows an elliptical fit to the peripheral radio structures, and the '+' marks its centre. The circles mark the positions of the two brightest cluster galaxies

Since $r_{\text {tot }}\left(v_{\text {sh }}\right)$ and $\phi\left(\mathcal{M}_{\text {sub }}\right)$ are shock velocity dependent, the simple scaling $K \propto$ $v_{\mathrm{sh}}^{2} \rho_{1}^{-2 / 3}$ is not valid. In CR-modified shocks $K_{\mathrm{mf}} \propto v_{\mathrm{sh}}^{v} \rho_{1}^{\left(1-\gamma_{\mathrm{g}}\right)}$ or $K_{\mathrm{mf}} \propto T^{v / a}$, where $v=2-\left(1+\gamma_{\mathrm{g}}\right) \xi+\sigma$. For the case of Alfvén wave heating the index $v$ is $\lesssim 1.25$ and $K_{\mathrm{mf}}$ is $\propto T^{0.8}$ assuming $\gamma_{\mathrm{g}}=5 / 3$. Recently Ponman et al. (2003) and Piffaretti et al. (2005) found that the dispersion in the observed cluster entropy profiles is smaller if an empirical relation $K \propto T^{0.65}$ is used instead of the standard $K \propto T$ (see also Pratt et al. 2006).

Consider the simple model of smooth accretion of cold gas through a strong accretion shock by Voit et al. (2003). The gas of velocity $v_{\text {ac }}$ accretes at a rate $\dot{M}_{g}$ through the shock at a radius $r_{\mathrm{ac}}$ where

$$
\dot{M}_{g}=4 \pi r_{\mathrm{ac}}^{2} \rho_{1} v_{\mathrm{ac}}, \quad v_{\mathrm{ac}}^{2}=2 G M \xi r_{\mathrm{ac}}^{-1}, \quad \xi=1-r_{\mathrm{ac}} / r_{\mathrm{ta}} .
$$

Here $M(t)$ is the cluster mass and $r_{\mathrm{ta}}$ is the matter turnaround radius. Then the entropy $K_{\mathrm{mf}}$ just behind the multi-fluid shock is expressed through $T_{i}^{(2)}\left(v_{\mathrm{ac}}\right)$ and $\rho_{2}=r_{\mathrm{tot}}\left(v_{\mathrm{ac}}\right) \rho_{1}$. In the Alfvén wave heating case $K_{\mathrm{mf}}(t) \propto(M t)^{(1+\sigma) / 3}$, instead of $K_{\mathrm{sf}}(t) \propto(M t)^{2 / 3}$ in the singlefluid regime. A multi-fluid shock results in a slower post-shock entropy production. As we have noted above, the regime of CR-shock compression depends on the plasma parameter $\beta$ in the infalling gas. The plasma parameter $\beta$ is currently poorly known because the intercluster magnetic fields are not well constrained. The effects of shock modifications are important for both the models of smooth accretion of cold gas and for accretion of hierarchical structures.

Preheating of accreting gas by different physical processes (e.g., due to early star formation in a protocluster region) was suggested by Evrard and Henry (1991), as a possible reason for the breaking of the scaling relations for pure gravitational cluster compression by Kaiser (1986). The observed high metallicity of clusters at different redshifts indicates that strong starburst activity was highly likely at some stage. The preheating produces some initial level of gas entropy ("entropy floor", see e.g., extensive simulations by Bialek et al. 2001; 
Borgani et al. 2001; Borgani et al. 2005). Multi-fluid strong shocks provide a natural alternative way of preheating accreting gas. The non-thermal components are essential for detailed modelling of global properties of X-ray clusters, including the mass-temperature and luminosity-temperature relations (Ostriker et al. 2005).

\section{Shocks in Large-Scale Structure}

Simulations of the cosmic large-scale structure (LSS) predict that about $40-50 \%$ of baryons at epoch $z<2$ could reside in the Warm-Hot Intergalactic Medium (WHIM) with temperatures $10^{5}-10^{7} \mathrm{~K}$ at moderate overdensities $\delta \sim 10$ (Cen and Ostriker 1999; Davé et al. 2001). The WHIM heating is due to shocks driven by gravitationally accelerated flows in the LSS structure formation scenario (e.g., Kang et al. 2007). The simulations demonstrate that the X-ray and ultraviolet O VI, O VII and O VIII lines and the H I Ly $\alpha$ line traces the low-density cosmic web filamentary structures. Intervening metal absorption systems of highly ionised $\mathrm{C}, \mathrm{N}, \mathrm{O}, \mathrm{Ne}$ in the soft X-ray spectra of bright AGN were suggested to trace the WHIM. The detection of shocked WHIM requires very sensitive UV and X-ray detectors, both for absorption and for emission processes (see, e.g., Kaastra et al. 2008; Richter et al. 2008-Chaps. 9 and 3, this issue). Dedicated future missions like the Cosmic Origin Spectrograph (COS), the X-Ray Evolving Universe Spectrometer (XEUS), Constellation- $X$ and the Diffuse Intergalactic Oxygen Surveyor (DIOS) will provide high resolution spectroscopy of the shocked WHIM. The WHIM ions of different charge states have highly non-equilibrium (anisotropic) initial states just behind a collisionless shock that relaxes to equilibrium states through Coulomb collisions. As was discussed above a strong collisionless shock could generate a spectrum of MHD-fluctuations. These MHDfluctuations can carry a substantial fraction of the shock ram pressure. The velocity fluctuations will result in non-thermal broadening of the lines, potentially important for simulations of emission/absorption spectra of the WHIM and observational data analysis. Specific features of collisionless shock heating of the WHIM ions are discussed in Bykov et al. (2008Chap. 8, this issue). In this paper we discuss only a few observations of clusters of galaxies.

\subsection{Evidence for Shocks in Galaxy Clusters}

Clusters of galaxies are believed to form within the hierarchical build up of the large scale structure of the Universe. Small objects collapse first and then merge in a complex manner to form larger and larger structures. Therefore, once in a while during their formation, clusters of galaxies undergo so called major merger events. In such events, proto-cluster structures of similar masses (typical ratios 1:10-1:1) are colliding with super sonic velocities (typically several $1000 \mathrm{~km} \mathrm{~s}^{-1}$ ). These merging events are a source of shocks and turbulence. They redistribute and amplify magnetic fields, and they are a source of acceleration of relativistic particles within the intracluster medium. Additionally, it is expected that the accretion of the diffuse, unprocessed (and therefore relatively cold) matter onto the DM node of the cosmic web creates a virialisation shock (also called accretion shock), which is expected to be located far in the cluster periphery (typically a few Mpc from the cluster centre for massive systems). Some examples of relevant cosmological simulations are presented by Dolag et al. (2008 - Chap. 15, this issue).

$\mathrm{X}$-ray observations, revealing the thermodynamical state of the intracluster medium are therefore the natural means for searching for the signatures of such non thermal phenomena. However, due to biases in the observational processes, caused by the complex temperature 
structure of the intracluster medium, such signatures are very hard to detect (for a more detailed discussion see Mazzotta et al. (2004) and references therein). Nevertheless, some detections of shocks in galaxy clusters have been revealed by high resolution Chandra and XMM-Newton observations. For a recent review see Markevitch and Vikhlinin (2007). One of the most spectacular examples of a merging galaxy clusters is the case of 1E 0657-56 (Markevitch et al. 2002).

The sensitivities of current X-ray instruments are not sufficient to map the state and structure of the intracluster medium in the periphery of galaxy clusters. However, the discovery of arc like radio emission in the periphery of some clusters (so called radio relics), are thought to trace shocks running through the intracluster medium. Spectacular examples are Abell 3667 (see also Ferrari et al. 2008-Chap. 6, this issue) or the radio relics in Abell 3376 recently discovered by Bagchi et al. (2006), see Fig. 8. Thereby, such radio observations are currently the only possibility to observe shocks outside the central regions of galaxy clusters. A more detailed discussion of numerical models of such radio relics is provided by Dolag et al. (2008-Chap. 15, this issue).

Shocks are also expected to trigger star formation, as indicated by numerical simulations (e.g., Bekki 1999), which will leave detectable imprints in the intracluster medium even long (several Gyr) after the shock passed through. Multiple supernova explosions in the star forming regions (superbubbles) will additionally produce copious small scale shocks and accelerate non-thermal particles (e.g., Bykov 2001). One of the tracers for these processes can be excess metallicity in the intracluster medium, produced by the enhanced star formation period (e.g., Schindler et al. 2005). Figure 9 shows the inferred metallicity map for Abell 3376, indicating previous merger activity of the cluster (see Bagchi et al. 2006).

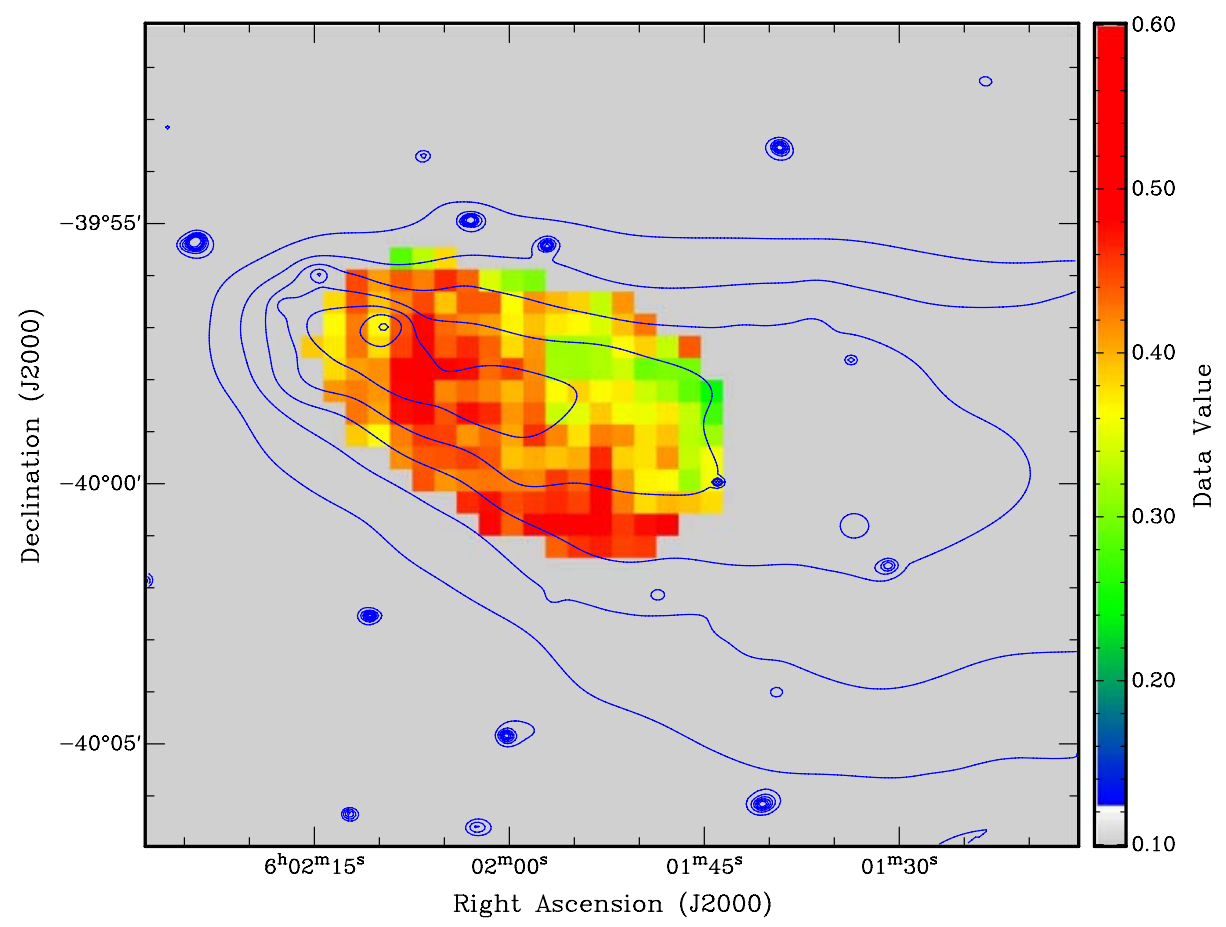

Fig. 9 Metallicity map of the intracluster gas in Abell 3376 (taken from Bagchi et al. 2006) 


\section{Summary}

Cosmological shocks convert a fraction of the energy of gravitationally accelerated flows to internal energy of the gas. They heat and compress the gas and can also accelerate energetic non-thermal particles and amplify magnetic fields. We discussed some specific features of cosmological shocks.

- The standard Rankine-Hugoniot relations based on the conservation laws for a steady single-fluid MHD shock allow to calculate the state of the fluid behind the shock once the upstream state and the shock strength are known. The coplanarity theorem for a plane ideal MHD shock states that the upstream and downstream bulk velocities, magnetic fields and the shock normal all lie in the same plane.

- Cosmological plasma shocks are likely to be collisionless as many other astrophysical shocks observed in the heliosphere and in supernova remnants. We review the basic plasma processes responsible for the microscopic structure of collisionless shocks.

- Collisionless shock heating of ions results in a non-equilibrium state just behind a very thin magnetic ramp region with a strongly anisotropic quasi-Maxwellian ion distributions. The possibility of collisionless heating of electrons by electromagnetic fluctuations in the magnetic ramp region depends on the extension of the fluctuation spectra to the electron gyro-scales, and could depend on the shock Mach number. Then the Coulomb equilibration processes are operating on the scales much larger than the collisionless shock width.

- Extended MHD shock waves propagating in turbulent media could accelerate energetic particles both by Fermi type acceleration in converging plasma flows and by DC electric field in quasi-perpendicular shocks. If the acceleration is efficient, then the strong shock could convert a substantial fraction (more than $10 \%$ ) of the power dissipated by the upstream bulk flow to energetic particles (cosmic rays). The compression ratio $r_{\text {tot }}$ at such a shock can be much higher, while the ion temperature behind the shock $\propto r_{\text {tot }}^{-2}$ and the post-shock entropy are lower, than that in a standard single fluid shock. The shock structure consists of an extended precursor and a viscous velocity jump (subshock) indicated in Fig. 5.

- Strong collisionless plasma shocks with an efficient Fermi acceleration of energetic particles could generate strong MHD waves in the upstream and downstream regions and strongly amplify the upstream magnetic fields. A distinctive feature of the shock is a predicted possibility of gas pre-heating in the far upstream region due to MHD wave dissipation, that can produce an extended filament of temperature $\gtrsim 0.1 \mathrm{keV}$.

- Shock waves both from the cosmic web formation processes and those due to cluster merging activity can play an important role in clusters of galaxies. Direct evidences for such shocks, as traced by radio relics and the temperature jumps in X-ray observations have been found only in a small number of clusters, and thus we need more observations.

Acknowledgements The authors thank ISSI (Bern) for support of the team "Non-virialised X-ray components in clusters of galaxies". A.M.B. thanks M.Yu. Gustov for his help with hybrid shock simulations. He acknowledges the RBRF grant 06-02-16844, a support from RAS Presidium Programmes. A support from NASA ATP (NNX07AG79G) is acknowledged.

\section{References}

E. Amato, P. Blasi, Mon. Not. R. Astron. Soc. 371, 1251 (2006)

W.I. Axford, E. Leer, G. Skadron, in International Cosmic Ray Conference, vol. 11 (1977), p. 132

J. Bagchi, F. Durret, G.B.L. Neto, S. Paul, Science 314, 791 (2006)

K. Bekki, Astrophys. J. 510, L15 (1999)

A.R. Bell, Mon. Not. R. Astron. Soc. 182, 147 (1978) 
A.R. Bell, Mon. Not. R. Astron. Soc. 353, 550 (2004)

A.R. Bell, P. Choi, A.E. Dangor, O. Willi, D.A. Bassett, Phys. Rev. A 38, 1363 (1988)

A.R. Bell, S.G. Lucek, Mon. Not. R. Astron. Soc. 321, 433 (2001)

E.G. Berezhko, V.K. Elshin, L.T. Ksenofontov, J. Exp. Theor. Phys. 82, 1 (1996)

J.J. Bialek, A.E. Evrard, J.J. Mohr, Astrophys. J. 555, 597 (2001)

R. Blandford, D. Eichler, Phys. Rep. 154, 1 (1987)

R.D. Blandford, J.P. Ostriker, Astrophys. J. 221, L29 (1978)

P. Blasi, Astropart. Phys. 21, 45 (2004)

S. Borgani, A. Diaferio, K. Dolag, S. Schindler, Space Sci. Rev. (2008). doi:10.1007/s11214-008-9317-4

S. Borgani, A. Finoguenov, S.T. Kay et al., Mon. Not. R. Astron. Soc. 361, 233 (2005)

S. Borgani, F. Governato, J. Wadsley et al., Astrophys. J. 559, L71 (2001)

D. Burgess, E.A. Lucek, M. Scholer et al., Space Sci. Rev. 118, 205 (2005)

A. Bykov, F.B.S. Paerels, V. Petrosian, Space Sci. Rev. (2008). doi:10.1007/s11214-008-9309-4

A.M. Bykov, Space Sci. Rev. 99, 317 (2001)

A.M. Bykov, Adv. Space Res. 36, 738 (2005)

A.M. Bykov, H. Bloemen, Y.A. Uvarov, Astron. Astrophys. 362, 886 (2000)

A.M. Bykov, I.N. Toptygin, Astrophys. Space Sci. 138, 341 (1987)

A.M. Bykov, Y.A. Uvarov, J. Exp. Theor. Phys. 88, 465 (1999)

C.L. Carilli, G.B. Taylor, Annu. Rev. Astron. Astrophys. 40, 319 (2002)

R. Cen, J.P. Ostriker, Astrophys. J. 514, 1 (1999)

R. Davé, R. Cen, J.P. Ostriker et al., Astrophys. J. 552, 473 (2001)

F. de Hoffmann, E. Teller, Phys. Rev. 80, 692 (1950)

K. Dolag, S. Borgani, S. Schindler, A. Diaferio, A. Bykov, Space Sci. Rev. (2008). doi:10.1007/ s11214-008-9316-5

B.T. Draine, C.F. McKee, Annu. Rev. Astron. Astrophys. 31, 373 (1993)

F. Durret, J.S. Kaastra, J. Nevalainen, T. Ohashi, N. Werner, Space Sci. Rev. (2008). doi:10.1007/ s11214-008-9313-8

D.C. Ellison, M.G. Baring, F.C. Jones, Astrophys. J. 473, 1029 (1996)

A.E. Evrard, J.P. Henry, Astrophys. J. 383, 95 (1991)

X. Fan, C.L. Carilli, B. Keating, Annu. Rev. Astron. Astrophys. 44, 415 (2006)

M.H. Farris, C.T. Russell, M.F. Thomsen, J.T. Gosling, J. Geophys. Res. 97, 19121 (1992)

C. Ferrari, F. Govoni, S. Schindler, A. Bykov, Y. Rephaeli, Space Sci. Rev. (2008). doi:10.1007/ s11214-008-9311-X

A. Finoguenov, U.G. Briel, J.P. Henry, Astron. Astrophys. 410, 777 (2003)

P. Ghavamian, J.M. Laming, C.E. Rakowski, Astrophys. J. 654, L69 (2007)

J. Giacalone, D.C. Ellison, J. Geophys. Res. 105, 12541 (2000)

F. Govoni, M. Murgia, L. Feretti et al., Astron. Astrophys. 430, L5 (2005)

M. Hoshino, N. Shimada, Astrophys. J. 572, 880 (2002)

F.C. Jones, D.C. Ellison, Space Sci. Rev. 58, 259 (1991)

F.C. Jones, J.R. Jokipii, M.G. Baring, Astrophys. J. 509, 238 (1998)

J.S. Kaastra, F.B.S. Paerels, F. Durret, S. Schindler, P. Richter, Space Sci. Rev. (2008). doi:10.1007/ s11214-008-9310-y

N. Kaiser, Mon. Not. R. Astron. Soc. 222, 323 (1986)

J.R. Kan, L.H. Lyu, M.E. Mandt, Space Sci. Rev. 57, 201 (1991)

H. Kang, D. Ryu, R. Cen, J.P. Ostriker, Astrophys. J. 669, 729 (2007)

C.F. Kennel, J.P. Edmiston, T. Hada, Geophys. Monogr. Ser. 34, 1 (1985)

P.A. Knight, T.J. Ponman, Mon. Not. R. Astron. Soc. 289, 955 (1997)

K.E. Korreck, T.H. Zurbuchen, S.T. Lepri, J.M. Raines, Astrophys. J. 659, 773 (2007)

G.F. Krymskii, Soviet Physics Doklady 22, 327 (1977)

L.D. Landau, E.M. Lifshitz, Fluid Mechanics (Pergamon, Oxford, 1959)

L.D. Landau, E.M. Lifshitz, Electrodynamics of Continuous Media, 2nd edn. (Pergamon, London, 1984)

B. Lembege, J. Giacalone, M. Scholer et al., Space Sci. Rev. 110, 161 (2004)

M.A. Malkov, L.O.C. Drury, Reports Prog. Phys. 64, 429 (2001)

M. Markevitch, A.H. Gonzalez, L. David et al., Astrophys. J. Lett. 567, L27 (2002)

M. Markevitch, A. Vikhlinin, Phys. Rep. 443, 1 (2007)

P. Mazzotta, E. Rasia, L. Moscardini, G. Tormen, Mon. Not. R. Astron. Soc. 354, 10 (2004)

F. Miniati, D. Ryu, H. Kang et al., Astrophys. J. 542, 608 (2000)

W.I. Newman, A.L. Newman, Y. Rephaeli, Astrophys. J. 575, 755 (2002)

C.A. Norman, D.B. Melrose, A. Achterberg, Astrophys. J. 454, 60 (1995)

J.P. Ostriker, P. Bode, A. Babul, Astrophys. J. 634, 964 (2005)

C. Pfrommer, V. Springel, T.A. Enßlin, M. Jubelgas, Mon. Not. R. Astron. Soc. 367, 113 (2006) 
R. Piffaretti, P. Jetzer, J.S. Kaastra, T. Tamura, Astron. Astrophys. 433, 101 (2005)

T.J. Ponman, A.J.R. Sanderson, A. Finoguenov, Mon. Not. R. Astron. Soc. 343, 331 (2003)

G.W. Pratt, M. Arnaud, E. Pointecouteau, Astron. Astrophys. 446, 429 (2006)

K.B. Quest, J. Geophys. Res. 93, 9649 (1988)

K.B. Quest, in AIP Conf. Ser., ed. by G.P. Zank, T.K. Gaisser. Particle Acceleration in Cosmic Plasmas, vol. 264, p. 349 (1992)

P. Richter, F.B.S. Paerels, J.S. Kaastra, Space Sci. Rev. (2008). doi:10.1007/s11214-008-9325-4

C.T. Russell, in AIP Conf. Ser, ed. by G. Li, G.P. Zank, C.T. Russell. The Physics of Collisionless Shocks, vol. 781, p. 3 (2005)

D. Ryu, H. Kang, E. Hallman, T.W. Jones, Astrophys. J. 593, 599 (2003)

R.Z. Sagdeev, Rev. Plasma Phys. 4, 23 (1966)

S. Schindler, W. Kapferer, W. Domainko et al., Astron. Astrophys. 435, L25 (2005)

H. Schmitz, S.C. Chapman, R.O. Dendy, Astrophys. J. 579, 327 (2002)

L. Spitzer, Physics of Fully Ionized Gases, 2nd edn. (Interscience, New York, 1962)

P. Tozzi, C. Norman, Astrophys. J. 546, 63 (2001)

B.T. Tsurutani, R.P. Lin, J. Geophys. Res. 90, 1 (1985)

A. Vladimirov, D.C. Ellison, A. Bykov, Astrophys. J. 652, 1246 (2006)

G.M. Voit, M.L. Balogh, R.G. Bower, C.G. Lacey, G.L. Bryan, Astrophys. J. 593, 272 (2003)

M.C. Weisskopf, J.P. Hughes, Astrophys. Update 2, 55 (2006)

D. Winske, N. Omidi, J. Geophys. Res. 101, 17287 (1996)

Y.B. Zel'dovich, Y.P. Raizer, Physics of Shock Waves and High-Temperature Hydrodynamic Phenomena (Academic, New York, 1967) 